Supporting Information

\title{
Preparation of both antipodes of enantiopure inherently chiral calix[4]crowns
}

\author{
Yu-Dong Cao, Jun Luo, Qi-Yu Zheng,* Chuan-Feng Chen, Mei-Xiang Wang,
}

\section{Zhi-Tang Huang*}

Table of contents

1 General Procedures.

2 Detailed experimental procedures and spectra of relative compounds $1 \mathrm{~b}, 2 \mathrm{~b}, 3 \mathrm{~b}, 4 \mathrm{~b}-1,4 \mathrm{~b}-2$, $(+)-3 b$ and $(-)-3 b$. 


\section{General Procedures.}

The solvents were purified as follows: DMF was dried with molecular sieves; DCM was freshly distilled from $\mathrm{CaH}_{2} . \mathrm{AlCl}_{3}$ was resublimation. ${ }^{1} \mathrm{H}$ and ${ }^{13} \mathrm{C} \mathrm{NMR}$ spectra were recorded at, respectively, 300 and 75 MHz. Flash column chromatography was carried out with silica gel (150-325 mesh). Thin-layer chromatographies (TLC) were performed with fluorescent silica gel F254 precoated on glasses. The dispatched reaction components were then visualized under UV light in order to be seperated. All reactions were performed under protection of inert atmosphere.

\section{Detailed experimental procedures and spectra of relative compounds $1 b, 2 b, 3 b, 4 b-1,4 b-2,(+)-3 b$ and (-)-3b.}

$\mathbf{1 b}$

Anhydrous $\mathrm{AlCl}_{3}\left(3.5 \mathrm{~g}, 5.1\right.$ equiv) was added to a solution of $1\left(\mathrm{R}_{1}=\mathrm{R}_{2}=t\right.$-Bu $)(3.85 \mathrm{~g}, 5.05 \mathrm{mmol})$ in dry toluene $(50 \mathrm{ml})$ and the mixture was stirred at $\mathrm{rt}$ for about $35 \mathrm{~min}$. Then $10 \% \mathrm{HCl}(50 \mathrm{ml})$ was added to quench the reaction. The organic phase was washed with water and dried over anhydrous $\mathrm{Na}_{2} \mathrm{SO}_{4}$. Compound $\mathbf{1 b}$ was isolated by column chromatography (petroleum ether: ethyl acetate, 6:1 v/v) as a white solid $(2.58 \mathrm{~g}$, yield 79\%); mp 189-191 ${ }^{\circ} \mathrm{C} ;{ }^{1} \mathrm{H}$ NMR $\left(\mathrm{CDCl}_{3}\right): \delta 8.94(\mathrm{~s}, 2 \mathrm{H}), 7.12(\mathrm{~s}, 2 \mathrm{H}), 7.01-6.95(\mathrm{~m}, 6 \mathrm{H}), 6.64(\mathrm{t}, 2 \mathrm{H}, J$ $=7.5 \mathrm{~Hz}), 4.64(\mathrm{~d}, 1 \mathrm{H}, J=12.2 \mathrm{~Hz}), 4.41(\mathrm{~d}, 1 \mathrm{H}, J=13.3 \mathrm{~Hz}), 4.30-3.90(\mathrm{~m}, 14 \mathrm{H}), 3.39$ and $3.37(2 \mathrm{~d}, 4 \mathrm{H}, J=$ 13.0Hz), $1.17(\mathrm{~s}, 18 \mathrm{H}) ;{ }^{13} \mathrm{C} \mathrm{NMR}\left(\mathrm{CDCl}_{3}\right): \delta 151.5,151.1,147.0,134.4,132.5,129.6,129.4,128.3,128.2$, 126.1, 125.9, 120.7, 75.7, 71.7, 70.1, 34.2, 32.5, 31.9, 31.4, 30.1; MALDI-TOF MS: m/z 673.3 ([M+Na $\left.]^{+}\right)$; IR (KBr): 3342, 1592, 1466; Anal. Calcd for $\mathrm{C}_{42} \mathrm{H}_{50} \mathrm{O}_{6}: \mathrm{C}, 77.51 ; \mathrm{H}, 7.74$. Found: C, 76.90; H, 7.67. 
$\mathbf{2 b}$ (racemic mixture)

To a stirred mixture of $\mathbf{1 b}(4 \mathrm{~g}, 6.15 \mathrm{mmol}), \mathrm{Cs}_{2} \mathrm{CO}_{3}(2 \mathrm{~g}, 1$ equiv) in dry DMF (400ml) was added $\mathrm{BrCH}_{2} \mathrm{COOEt}\left(0.8 \mathrm{ml}, 1.2\right.$ equiv) and the reaction mixture was heated at $60{ }^{\circ} \mathrm{C}$ overnight under nitrogen. Then a small amount of $10 \% \mathrm{HCl}$ was added to quench the reaction. The solvent was removed under reduced pressure and the residue was partitioned between water and $\mathrm{CH}_{2} \mathrm{Cl}_{2}$. The organic layer was dried over anhydrous $\mathrm{Na}_{2} \mathrm{SO}_{4}$. Compound $\mathbf{2} \mathbf{b}$ was isolated by column chromatography (petroleum ether: ethyl acetate, 6:1 v/v) as a white solid (3.46g, yield 76\%); mp 168-170 ${ }^{\circ} \mathrm{C} ;{ }^{1} \mathrm{H} \mathrm{NMR}\left(\mathrm{CDCl}_{3}\right): \delta 7.18(\mathrm{~d}, 1 \mathrm{H}, J=2.0 \mathrm{~Hz})$, 7.11-7.05 (m, 3H), $6.76(\mathrm{t}, 1 \mathrm{H}, J=7.4 \mathrm{~Hz}), 6.45(\mathrm{~d}, 2 \mathrm{H}, J=2.5 \mathrm{~Hz}), 6.27(\mathrm{~s}, 3 \mathrm{H}), 5.12(\mathrm{~s}, 1 \mathrm{H}), 4.60,4.53,4.50$, $4.46(4 \mathrm{~d}, 4 \mathrm{H}, J=13.3 \mathrm{~Hz}), 4.38-3.70(\mathrm{~m}, 14 \mathrm{H}), 4.02(\mathrm{q}, 2 \mathrm{H}), 3.31,3.27,3.19,3.16(4 \mathrm{~d}, 4 \mathrm{H}, J=13.0 \mathrm{~Hz})$, 1.36 and $0.83(2 \mathrm{~s}, 18 \mathrm{H}), 1.31(\mathrm{t}, 3 \mathrm{H}, J=7.1 \mathrm{~Hz}) ;{ }^{13} \mathrm{C} \mathrm{NMR}\left(\mathrm{CDCl}_{3}\right): \delta 169.3,154.7,154.0,153.3,150.5$, $146.3,145.9,136.2,135.9,133.3,133.0,131.1,130.8,129.7,128.6,128.3,127.8,127.7,127.6,126.2,125.9$, $125.8,124.5,123.3,119.0,74.8,72.6,72.1,71.8,70.1,69.8,69.3,61.1,34.2,33.8,31.8,31.3,31.1$, 30.3,14.3; MALDI-TOF MS: m/z $759.6\left([\mathrm{M}+\mathrm{Na}]^{+}\right)$; IR (KBr): 3526, 1759, 1589, 1480, 1458; Anal. Calcd for $\mathrm{C}_{46} \mathrm{H}_{56} \mathrm{O}_{8}:$ C, 74.97; H, 7.66. Found: C, 74.80; H, 7.71.

\section{3b (racemic mixture)}

To a solution of $\mathrm{NaOH}\left(54 \mathrm{mg}\right.$, 5equiv) in $\mathrm{EtOH} / \mathrm{H}_{2} \mathrm{O}(10 \mathrm{ml} / 10 \mathrm{ml})$ was added $2 \mathbf{b}(200 \mathrm{mg}, 0.27 \mathrm{mmol})$ and the reaction mixture was refluxed for $3 \mathrm{~h}$. After removing the solvent under reduced pressure, $10 \% \mathrm{HCl}(15 \mathrm{ml})$ was added. The precipitate, after being filtered and washed with water, was crystallized from $\mathrm{CH}_{2} \mathrm{Cl}_{2} / \mathrm{MeOH}$ to afford pure compound $\mathbf{3 b}$ as a colorless crystal (160mg, yield 77\%); mp125-127 ${ }^{\circ} \mathrm{C} ;{ }^{1} \mathrm{H} \mathrm{NMR}\left(\mathrm{CDCl}_{3}\right): \delta$ 
8.31 (s, 1H), 7.11-6.73 (m, 10H), 4.91, 4.86, 4.39, 4.35 (4d, 4H, J = 14.2 Hz), 4.28-3.90 (m, 14H), 3.49, 3.39, 3.38, $3.34(4 \mathrm{~d}, 4 \mathrm{H}, J=13.8 \mathrm{~Hz}), 1.22$ and $1.17(2 \mathrm{~s}, 18 \mathrm{H}) ;{ }^{13} \mathrm{C} \mathrm{NMR}\left(\mathrm{CDCl}_{3}\right): \delta 170.8,153.0,152.5,152.2$, $149.9,147.5,146.1,135.1,134.6,133.5,133.2,131.4,129.5,128.9,128.8,128.7,128.6,128.5,128.2,126.1$, $126.0,125.6,125.4,125.0,120.1,75.2,74.4,71.5,70.4,70.3,70.2,69.2,34.1,32.5,31.8,31.4,31.3,29.8$ MALDI-TOF MS: m/z $731.4\left([\mathrm{M}+\mathrm{Na}]^{+}\right)$; IR (KBr): 3444, 1739, 1480, 1463; Anal. Calcd for $\mathrm{C}_{44} \mathrm{H}_{52} \mathrm{O}_{8}: \mathrm{C}_{\text {, }}$ 74.55; H, 7.39. Found: C, 74.48; H, 7.24.

\section{4b-1 and 4b-2 (diastereomers)}

A solution of 3b (1.1g, 1.55mmol), (S)-BINOL (0.44g, 1equiv), DCC (320mg, 1equiv) and DMAP (20mg, 0.1 equiv) in $\mathrm{CH}_{2} \mathrm{Cl}_{2}(110 \mathrm{ml})$ was stirred at $\mathrm{rt}$ for $5 \mathrm{~h}$. During this period, a large amount of insoluble DCU was formed which was removed by filtration. After removal of solvent, the residue was purified by column chromatography (petroleum ether: ethyl acetate, 6:1 v/v) to afford $\mathbf{4 b - 1}$ and $\mathbf{4 b - 2}$ as a white solid of diastereomeric mixture (1.2g in total, yield 78\%) which was then subject to preparative TLC using $\mathrm{CHCl}_{3} /$ ethyl acetate (5:1, v/v) as eluent. The two dispatched bands (UV visible) were collected respectively and soaked in acetone for several hours (decomposed slightly). Then each was eluted with acetone through a short column chromatography. After evaporation of acetone, each was further purified by column chromatography (petroleum ether: ethyl acetate, 6:1 v/v) to afford $\mathbf{4 b - 1}$ and $\mathbf{4 b - 2}$ as a pure enantiomer respectively (yield $25 \%$ each).

\section{4b-1:}

mp 141-143 ${ }^{\circ} \mathrm{C} ;{ }^{1} \mathrm{H}$ NMR $\left(\mathrm{CDCl}_{3}\right): \delta 8.05(\mathrm{~d}, 1 \mathrm{H}, J=8.9 \mathrm{~Hz}), 7.96(\mathrm{~d}, 1 \mathrm{H}, J=8.2 \mathrm{~Hz}), 7.82-7.78(\mathrm{~m}, 2 \mathrm{H})$, $7.47(\mathrm{~d}, 2 \mathrm{H}, J=8.8 \mathrm{~Hz}), 7.34-7.15(\mathrm{~m}, 6 \mathrm{H}), 7.15-7.02(\mathrm{~m}, 4 \mathrm{H}), 6.77(\mathrm{t}, 1 \mathrm{H}, J=7.5 \mathrm{~Hz}), 6.21(\mathrm{~s}, 2 \mathrm{H}), 6.20(\mathrm{~s}$, 
3H), 4.90 (s, 1H), 4.49, 4.33, 4.25, 4.14 (4d, 4H, $\left.J=13.4 \mathrm{~Hz}, \mathrm{ArCH}_{2} \mathrm{Ar}\right), 3.34,3.13,3.00,2.84$ (4d, 4H, $J$ $\left.=13.7 \mathrm{~Hz}, \mathrm{ArCH}_{2} \mathrm{Ar}\right), 4.42-3.70(\mathrm{~m}, 14 \mathrm{H}), 1.37$ and $0.80(2 \mathrm{~s}, 18 \mathrm{H}) ;{ }^{13} \mathrm{C} \mathrm{NMR}\left(\mathrm{CDCl}_{3}\right): \delta 167.9,154.2,153.6$, 153.0, 152.1, 150.1, 147.1, 146.0, 145.6, 135.8, 135.7, 133.8, 133.4, 133.1, 132.8, 132.5, 132.1, 130.8, 130.5, $130.2,130.0,129.5,128.6,128.4,128.2,128.1,127.8,127.5,127.4,127.0,126.5,126.0,125.9,125.8,125.7$, $125.6,124.4,124.2,124.1,123.2,123.1,121.4,118.8,118.2,114.0,74.2,72.1,71.3,70.9,69.7,69.1,68.1$, 34.0, 33.5, 31.5, 31.0, 30.8, 30.6, 30.5, 30.0; MALDI-TOF MS: m/z 999.5 ([M+Na $\left.]^{+}\right)$; IR (KBr): 3467, 1784, 1622, 1595, 1510, 1462; Anal. Calcd for $\mathrm{C}_{64} \mathrm{H}_{64} \mathrm{O}_{9} \square \mathrm{C}, 78.66$; H, 6.60. Found: C, 78.88; H, 6.85.

\section{4b-2:}

mp 140-142 ${ }^{\circ} \mathrm{C} ;{ }^{1} \mathrm{H}$ NMR $\left(\mathrm{CDCl}_{3}\right): \delta 8.08(\mathrm{~d}, 1 \mathrm{H}, J=8.9 \mathrm{~Hz}), 7.98(\mathrm{~d}, 1 \mathrm{H}, J=8.2 \mathrm{~Hz}), 7.85-7.81(\mathrm{~m}, 2 \mathrm{H}$,$) ,$ $7.52(\mathrm{~d}, 2 \mathrm{H}, J=8.6 \mathrm{~Hz}), 7.36-7.21(\mathrm{~m}, 6 \mathrm{H}), 7.11(\mathrm{~s}, 1 \mathrm{H}), 7.06(\mathrm{~d}, 3 \mathrm{H}, J=7.7 \mathrm{~Hz}), 6.74(\mathrm{t}, 1 \mathrm{H}, J=7.5 \mathrm{~Hz}), 6.43$ (s, 2H), $6.26(\mathrm{~m}, 3 \mathrm{H}), 4.98(\mathrm{~s}, 1 \mathrm{H}), 4.56,4.24,4.22,4.19\left(4 \mathrm{~d}, 4 \mathrm{H}, J=13.5 \mathrm{~Hz}, \mathrm{ArCH}_{2} \mathrm{Ar}\right), 4.36-3.65$ (m, $\left.14 \mathrm{H}\right)$, 3.31, 3.20, 3.08, $2.94(4 \mathrm{~d}, 4 \mathrm{H}, J=13.5 \mathrm{~Hz}, \mathrm{ArCH} 2 \mathrm{Ar}), 1.41$ and $0.81(2 \mathrm{~s}, 18 \mathrm{H}) ;{ }^{13} \mathrm{C} \mathrm{NMR}\left(\mathrm{CDCl}_{3}\right): \delta 167.5$, 153.6, 153.5, 153.0, 152.0, 150.1, 147.1, 145.9, 145.7, 136.0, 135.4, 133.4, 133.3, 132.9, 132.7, 132.5, 132.0, 131.0, 130.1, 130.0, 129.9, 129.3, 128.5, 128.4, 128.1, 128.0, 127.7, 127.5, 127.3, 126.9, 126.4, 126.0, 125.9, $125.8,125.5,125.4,125.3,124.4,124.3,124.0,123.2,121.4,118.6,118.2,114.0,74.1,71.7,71.6,70.8,69.4$, 69.1, 68.2 , 34.0, 33.6, 31.2, 30.8, 30.7, 30.5, 30.3, 30.1; MALDI-TOF MS: m/z 999.5 ([M+Na] $\left.]^{+}\right)$; IR (KBr): 3531, 1783, 1621, 1597; Anal. Calcd for $\mathrm{C}_{64} \mathrm{H}_{64} \mathrm{O}_{9} \square \mathrm{C}, 78.66$; H, 6.60. Found: C, 78.78; H, 6.85.

\section{(+)-3b and (-)-3b (enantiomeric pure)}

Hydrolyzing 4b-1 and 4b-2 according to the method of preparing 3 afford (+)-3b and (-)-3b as a white solid respectively (yield $80 \%$ each). Both have similar mp, ${ }^{1} \mathrm{HNMR}$ and ${ }^{13} \mathrm{CNMR}$ with racemic mixture $3 \mathbf{b}$. 
(+)-3b

mp 120-122 ${ }^{\circ} \mathrm{C} ;{ }^{1} \mathrm{H}$ NMR (CDCl3): $\delta 8.10(\mathrm{~s}, 1 \mathrm{H}), 7.14-6.70(\mathrm{~m}, 10 \mathrm{H}), 4.83,4.79,4.39,4.36(4 \mathrm{~d}, 4 \mathrm{H}, J$ $\left.=13.0 \mathrm{~Hz}, \mathrm{ArCH}_{2} \mathrm{Ar}\right), 4.27-3.84(\mathrm{~m}, 14 \mathrm{H}), 3.43,3.34\left(2 \mathrm{~d}, 4 \mathrm{H}, J=13.1 \mathrm{~Hz}, \mathrm{ArCH}_{2} \mathrm{Ar}\right), 1.25$ and $1.18(2 \mathrm{~s}$, 18H); MALDI-TOF MS: m/z $731.4\left([\mathrm{M}+\mathrm{Na}]^{+}\right) ; \mathrm{IR}(\mathrm{KBr}): 3430,1737,1480,1462 ;[\mathrm{a}]_{\mathrm{D}}+20^{\circ}\left(\mathrm{c} 0.5, \mathrm{CHCl}_{3}\right)$; Anal. Calcd for $\mathrm{C}_{44} \mathrm{H}_{52} \mathrm{O}_{8}, 0.5 \mathrm{H}_{2} \mathrm{O}$ : C, 73.61; H, 7.44. Found: C, 73.82; H, 7.94.

$(-)-3 b$

mp 120-122 ${ }^{\circ} \mathrm{C} ;{ }^{1} \mathrm{H}$ NMR (CDCl3): $\delta 8.10(\mathrm{~s}, 1 \mathrm{H}), 7.13-6.73(\mathrm{~m}, 10 \mathrm{H}), 4.86,4.82,4.40,4.36(4 \mathrm{~d}, 4 \mathrm{H}, J$ $\left.=13.1 \mathrm{~Hz}, \mathrm{ArCH}_{2} \mathrm{Ar}\right), 4.28-3.85(\mathrm{~m}, 14 \mathrm{H}), 3.46,3.39,3.38,3.34\left(4 \mathrm{~d}, 4 \mathrm{H}, J=14.3 \mathrm{~Hz}, \mathrm{ArCH}_{2} \mathrm{Ar}\right), 1.24$ and $1.13(2 \mathrm{~s}, 18 \mathrm{H}) ;$ MALDI-TOF MS: m/z $731.5\left([\mathrm{M}+\mathrm{Na}]^{+}\right) ; \mathrm{IR}(\mathrm{KBr}): 3430,1737,1480,1463 ;[\mathrm{a}]_{\mathrm{D}}-20^{\circ}(\mathrm{c} 0.5$, $\mathrm{CHCl}_{3}$ ); Anal. Calcd for $\mathrm{C}_{44} \mathrm{H}_{52} \mathrm{O}_{8}, 0.5 \mathrm{H}_{2} \mathrm{O}: \mathrm{C}, 73.61 ; \mathrm{H}, 7.44$. Found: C, 73.98; H, 7.83. 